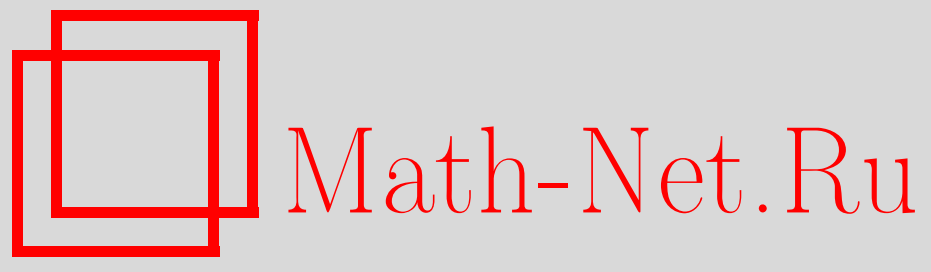

Е. Ю. Бунькова, Дифференциально-геометрическая структура универсального расслоения эллиптических кривых, УМH, 2011, том 66, выпуск 4, 185-186

DOI: https://doi.org/10.4213/rm9436

Использование Общероссийского математического портала Math-Net.Ru подразумевает, что вы прочитали и согласны с пользовательским соглашением http://www . mathnet.ru/rus/agreement

Параметры загрузки:

IP: 54.172 .240 .79

26 апреля 2023 г., 16:14:53

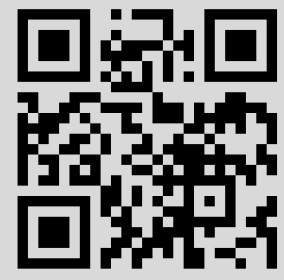




\section{Дифференциально-геометрическая структура универсального расслоения эллиптических кривых}

\section{Е. Ю. Бунькова}

Слоем универсального расслоения $\mathcal{E}_{1} \rightarrow \mathbb{C}^{2}$ эллиптических кривых является кривая $V$ с аффинной частью $\left\{(x, y) \mid y^{2}=4 x^{3}-g_{2} x-g_{3}\right\}$. Поля на базе $\mathbb{C}^{2}$ с координатами $\left(g_{2}, g_{3}\right)$ (см. [1]) $L_{0}=4 g_{2} \partial_{g_{2}}+6 g_{3} \partial_{g_{3}}, L_{2}=6 g_{3} \partial_{g_{2}}+\left(g_{2}^{2} / 3\right) \partial_{g_{3}}$, где $\partial_{g_{2}}=\partial / \partial g_{2}$, $\partial_{g_{3}}=\partial / \partial g_{3}$, с коммутатором $\left[L_{0}, L_{2}\right]=2 L_{2}$ образуют базис в пространстве векторных полей, касающихся дискриминанта $\Delta=\left\{\left(g_{2}, g_{3}\right) \mid g_{2}^{3}=27 g_{3}^{2}\right\}$. Им соответствует связность Гаусса-Манина $\nabla$ на $\mathcal{E}_{1}$ :

$$
\nabla_{L_{n}}\left(d_{1}, \quad d_{2}\right)=\left(d_{1}, \quad d_{2}\right) \Gamma_{n}, \quad n=0,2,
$$

где $d_{1}=\frac{x d x}{y}, d_{2}=\frac{d x}{y}, \Gamma_{0}=\left(\begin{array}{cc}1 & 0 \\ 0 & -1\end{array}\right), \Gamma_{2}=\frac{1}{12}\left(\begin{array}{cc}0 & 12 \\ -g_{2} & 0\end{array}\right)$.

Выбрав $d_{1}$ и $d_{2}$ в качестве базисных векторов в слое касательного расслоения к кривой, мы получаем действие $\nabla_{L_{n}}$ на координатах $A^{\top}=\left(A_{1}, A_{2}\right)$ голоморфного дифференциала общего вида $A_{1} d_{1}+A_{2} d_{2}$ на проколотой в $\infty$ эллиптической кривой.

Лемма. Имеет место формула $\nabla_{\left[L_{0}, L_{2}\right]} A=\left[\nabla_{L_{0}}, \nabla_{L_{2}}\right] A$, т.е. связность ГауссаМанина расслоения $\mathcal{E}_{1}$ является плоской.

Связность $\nabla$ согласована с симметричной кометрикой $G=\left(g^{i, j}\right)$, если матрица $G$ удовлетворяет совместной системе дифференциальных уравнений в неголономном репере $L_{k} G=\Gamma_{k}^{\top} G+G \Gamma_{k}, k=0,2$.

Положим $z=g_{2}^{3} /\left(27 g_{3}^{2}\right), t^{12}=g_{2}^{3}-27 g_{3}^{2}, \xi(z)=-2 \sqrt{3} z^{2 / 3}(z-1)^{5 / 6}$.

Tеорема 1. В координатах $t, z$ поля $L_{0}$ и $L_{2}$ имеют вид $L_{0}=t \partial_{t} u L_{2}=t^{2} \xi(z) \partial_{z}$.

Положим $g^{1,1}=-\frac{t^{2} \xi(z)}{2(z-1)} f_{1,1}(z), g^{1,2}=f_{1,2}(z), g^{2,2}=-\frac{2 z(z-1)}{t^{2} \xi(z)} f_{2,2}(z)$.

Теорема 2. Связность $\nabla$ согласована с $G$, если

$-2 \operatorname{det} M \frac{d}{d z} F=M F, \quad$ əде $F=\left(\begin{array}{l}f_{1,1}(z) \\ f_{1,2}(z) \\ f_{2,2}(z)\end{array}\right), M=\frac{1}{12}\left(\begin{array}{ccc}12(4-3 z) & 2 & 0 \\ -36 z & 0 & z \\ 0 & -72 & 12(2-3 z)\end{array}\right)$.

СледСтвиЕ. Функция $\operatorname{det} G=z f_{1,1}(z) f_{2,2}(z)-f_{1,2}(z)^{2}$ локалъно постоянна.

Теорема 3. 1) В области $|z|<1$ симметричные кометрики на $\mathbb{C}^{2}$, согласованные со связностью Гаусса-Манина на $\mathcal{E}_{1}$, задаются абсолютно сходящимися рядами

$$
f_{1,1}(z)=\sum_{n=0}^{\infty} c_{n}^{1,1} z^{n}, \quad f_{1,2}(z)=\sum_{n=0}^{\infty} c_{n}^{1,2} z^{n}, \quad f_{2,2}(z)=\sum_{n=0}^{\infty} c_{n}^{2,2} z^{n}
$$

где $c_{-1}^{i, j}=0, c_{0}^{1,2}-$ свободный параметр, а $c_{n}^{i, j}$ задаются рекуррентно:

$$
\begin{gathered}
c_{n}^{1,1}=\frac{18(2 n-1) c_{n-1}^{1,1}-c_{n}^{1,2}}{12(3 n+2)}, \quad c_{n}^{1,2}=\frac{72(n-1) c_{n-1}^{1,2}+36 c_{n-1}^{1,1}-c_{n-1}^{2,2}}{72 n}, \\
c_{n}^{2,2}=\frac{3(2 n-1) c_{n-1}^{2,2}+6 c_{n}^{1,2}}{2(3 n+1)} .
\end{gathered}
$$

2) В области $|z|>1$ симметричные кометрики на $\mathbb{C}^{2}$, согласованные со связностью Гаусса-Манина на $\mathcal{E}_{1}$, задаются абсолютно сходящимися рядами

$$
z f_{1,1}(z)=\sum_{n=0}^{\infty} b_{n}^{1,1} z^{-n}, \quad f_{1,2}(z)=\sum_{n=0}^{\infty} b_{n}^{1,2} z^{-n}, \quad z f_{2,2}(z)=\sum_{n=0}^{\infty} b_{n}^{2,2} z^{-n},
$$


где $b_{-1}^{i, j}=0, b_{0}^{1,2}-$ свободный параметр, а $b_{n}^{i, j}$ задаются рекуррентно:

$$
\begin{gathered}
b_{n}^{1,1}=\frac{12(3 n-2) b_{n-1}^{1,1}-b_{n}^{1,2}}{18(2 n+1)}, \quad b_{n}^{1,2}=\frac{72(n-1) b_{n-1}^{1,2}+36 b_{n-1}^{1,1}-b_{n-1}^{2,2}}{72 n}, \\
b_{n}^{2,2}=\frac{2(3 n-1) b_{n-1}^{2,2}+6 b_{n}^{1,2}}{3(2 n+1)} .
\end{gathered}
$$

Следствие. При $|z|<1$ имеем $\operatorname{det} G=-\left(c_{0}^{1,2}\right)^{2}$, a $п р u|z|>1$ имеем $\operatorname{det} G=-\left(b_{0}^{1,2}\right)^{2}$.

Приведем результаты, которые были получены при распространении описанных выше результатов на случай рода 2 . Слоем универсального расслоения $\mathcal{E}_{2} \rightarrow \mathbb{C}^{4}$ кривых рода 2 является кривая $V$ с аффинной частью $\left\{(x, y) \mid y^{2}=4 x^{5}+\lambda_{4} x^{3}+\lambda_{6} x^{2}+\right.$ $\left.\lambda_{8} x+\lambda_{10}\right\}$. В [2] описаны векторные поля, касающиеся дискриминанта соответствующего семейства кривых, и построена градуированная полиномиальная алгебра Ли $\mathcal{L}_{\mathcal{A}}$ (см. [3]) с образующими размерностей $0,2,4,6$ над $\mathcal{A}=\mathbb{C}\left[\lambda_{4}, \lambda_{6}, \lambda_{8}, \lambda_{10}\right], \operatorname{deg} \lambda_{k}=k$.

Tеорема 4. В $\mathcal{L}_{\mathcal{A}}$ существует базис $L=\left(L_{0}, L_{2}, L_{4}, L_{6}\right)^{\top}, \operatorname{deg} L_{k}=k$, скоммутачионными соотношениями $\left[L_{0}, L_{k}\right]=k L_{k}, k=2,4,6, u$

$$
\begin{gathered}
{\left[L_{2}, L_{4}\right]=-\frac{1}{4} \lambda_{4} L_{2}+2 L_{6}, \quad\left[L_{2}, L_{6}\right]=\frac{1}{20} \lambda_{6} L_{2}-\frac{1}{5} \lambda_{4} L_{4},} \\
{\left[L_{4}, L_{6}\right]=\frac{3}{10} \lambda_{8} L_{2}-\frac{1}{5} \lambda_{6} L_{4}+\frac{1}{20} \lambda_{4} L_{6}}
\end{gathered}
$$

и действием на $\mathcal{A}: L=T\left(\partial_{\lambda_{4}}, \partial_{\lambda_{6}}, \partial_{\lambda_{8}}, \partial_{\lambda_{10}}\right)^{\top}$, где

$$
T=\left(\begin{array}{cccc}
4 \lambda_{4} & 6 \lambda_{6} & 8 \lambda_{8} & 10 \lambda_{10} \\
6 \lambda_{6} & 8 \lambda_{8}-\frac{3}{5} \lambda_{4}^{2} & 10 \lambda_{10}-\frac{2}{5} \lambda_{4} \lambda_{6} & -\frac{1}{5} \lambda_{4} \lambda_{8} \\
8 \lambda_{8}-\frac{3}{10} \lambda_{4}^{2} & 10 \lambda_{10}-\frac{17}{20} \lambda_{4} \lambda_{6} & \frac{2}{5} \lambda_{4} \lambda_{8}-\frac{3}{5} \lambda_{6}^{2} & \frac{3}{4} \lambda_{4} \lambda_{10}-\frac{3}{10} \lambda_{6} \lambda_{8} \\
10 \lambda_{10}-\frac{1}{10} \lambda_{4} \lambda_{6} & -\frac{1}{5} \lambda_{4} \lambda_{8}-\frac{3}{20} \lambda_{6}^{2} & \frac{3}{2} \lambda_{4} \lambda_{10}-\frac{1}{2} \lambda_{6} \lambda_{8} & \frac{3}{4} \lambda_{6} \lambda_{10}-\frac{2}{5} \lambda_{8}^{2}
\end{array}\right) .
$$

СледствиЕ. Операторы $L_{2}, L_{4}, L_{6}$ порождают подалгебру $\widehat{\mathcal{L}}_{\mathcal{A}}$ в $\mathcal{L}_{\mathcal{A}}$ с коммутационными соотношениями над кольцом $\widehat{\mathcal{A}}=\mathbb{C}\left[\lambda_{4}, \lambda_{6}, \lambda_{8}\right]$.

ПредложениЕ. Не существует градуированной полиномиалъной алгебры Ли $\widehat{\mathcal{L}}_{\widehat{\mathcal{A}}}$ с образуюшими $\widehat{L}_{2}, \widehat{L}_{4}, \widehat{L}_{6}$ над $\widehat{\mathcal{A}}$ и коммутационными соотношениями (1).

ДоказАтельСтво. Пусть $\left[\widehat{L}_{i}, \widehat{L}_{j}\right]=\sum c_{i, j}^{k} \widehat{L}_{k}$ и $\widehat{L}_{i} \lambda_{q}=v_{i, q}$. Обобщенные соотношения Якоби (см. [3]) в нашем случае эквивалентны следующим:

$$
\begin{gathered}
v_{2,4}=6 \lambda_{6}, \quad v_{4,4}=v_{2,6}+\frac{3}{10} \lambda_{4}^{2}, \quad v_{4,6}=6 v_{2,8}-5 v_{6,4}+\frac{21}{20} \lambda_{4} \lambda_{6}, \\
\sum_{q=2,4,6} c_{i, j}^{q} v_{q, k}=\sum_{q=4,6,8}\left(v_{i, q} \frac{\partial v_{j, k}}{\partial \lambda_{q}}-v_{j, q} \frac{\partial v_{i, k}}{\partial \lambda_{q}}\right) .
\end{gathered}
$$

Подставляя выражения для $v_{i, k}$, где $\operatorname{deg} v_{i, k}=i+k$, как полиномы от $\lambda_{q}$, приходим к несовместной системе уравнений.

\section{Список литературы}

[1] G. Frobenius, L. Stickelberger, Kronecker J., 92 (1882), 311-337. [2] В. М. Бухштабер, Д. В. Лейкин, Функи. анализ и его прил., 42:4 (2008), 24-36. [3] В. М. Бухштабер, Д. В. Лейкин, Функи. анализ и его прил., 36:4 (2002), 18-34.

\section{Е. Ю. Бунькова (Е. Yu. Bun'kova)}

Математический институт им. В. А. Стеклова РАН E-mail: bunkova@mi.ras.ru
Представлено В. М. Бухштабером Принято редколлегией 07.07.2011 